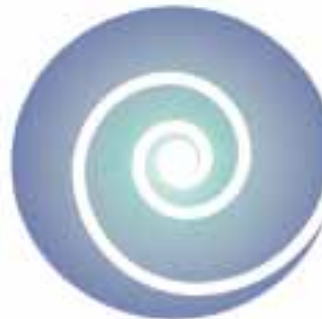

\title{
Measuring the Effects of Massage on Exercise Performance and Cardiopul- monary Response in Children With and Without Heart Disease: A Pilot Study
}

Shay Beider, MPH, LMT*; Karen T. Boulanger, MS, CMT*; Milind Joshi, BS; Yann Ping Pan, MD; Ruey-Kang R. Chang, MD, MPH†

*Integrative Touch for Kids, Tucson, AZ, USA; 'Department of Pediatrics, Harbor-UCLA Medical Center, Torrance, CA, USA.

Background: Congenital heart disease, a common and serious birth defect, affects 8 per 1000 live-born infants. Decreased exercise capacity and development of obesity is common in this population. These children may benefit from therapies, such as massage therapy, that could enhance cardiovascular and skeletal muscle function when they exercise.

Purpose: A pilot study conducted at the pediatric cardiology clinic of the Mattel Children's Hospital of the University of California-Los Angeles examined the safety and feasibility of measuring the effects of pre-exercise massage on exercise performance and cardiopulmonary response in children with and without heart disease.

Participants and Methods: Sixteen children (mean age: $9.2 \pm 2.2$ years) participated in the study. Ten participants had various forms of heart disease, and six children were healthy.

A female certified massage therapist with specialized training in pediatric massage provided a 30-minute massage to the participants. Using a standard protocol, each participant underwent two exercise tests: one test with and one without pre-exercise massage.

Heart rate, blood pressure, and oxygen uptake $\left(\mathrm{VO}_{2}\right)$ were measured in the participants.

Results: All recruited participants completed the study. No adverse events occurred during any of the exercise tests or massage sessions. Measurements during exercise with or without a preceding massage were compared, and the pre-exercise massage condition yielded a significantly higher heart rate and higher minute ventilation. Measurements during exercise in children with heart disease and in healthy participants showed no significant differences in peak heart rate, blood pressure, peak $\mathrm{VO}_{2}$, peak work rate, minute ventilation, or respiratory quotient.

Conclusions: In this study, peak heart rate, peak $\mathrm{VO}_{2}$, and peak minute ventilation were higher when children received a massage before exercise testing. Larger studies will be needed to investigate the strength of this finding. Future studies should include measurements of anxiety and psychological factors in addition to cardiopulmonary measures.

KEYWORDS: Comfort, cardiac, CAM, integrative medicine, pediatric

\section{INTRODUCTION}

Congenital heart disease (CHD), a common and serious birth defect, affects 8 per 1000 live-born infants ${ }^{(1)}$. Although advances in pediatric cardiology and cardiac surgery have greatly improved outcomes in infants with CHD, recent data indicate that CHD still accounts for $21 \%$ of all deaths in the first month of life and $40 \%$ of deaths from congenital malformations ${ }^{(2)}$. Most infants with CHD can undergo successful surgical repair or palliation. Although most children with CHD live to adulthood, decreased exercise capacity and development of obesity is common in this population ${ }^{(3)}$. These children may benefit from therapies that enhance cardiovascular and skeletal muscle function when they exercise. One potential intervention is massage therapy (MT). Our pilot study therefore evaluated the feasibility and safety of measuring the effects of pre-exercise massage on exercise performance and cardiopulmonary response in children with and without heart disease.

Pediatric MT is a growing field. An increasing number of MT studies are being performed in children and adolescents, including reviews, randomized controlled trials, evaluation studies, and descriptive case studies. To date, more than 24 randomized controlled trials of MT for children between the ages of 2 and 19 years have been conducted ${ }^{(4)}$. These studies demonstrate positive changes in depression and negative mood, and in air flow in children with pulmonary disorders. Notably, MT has shown a considerable effect on state and trait anxiety levels in children. This effect is strong and consistent with similar effects found in adult studies.

In adults, MT has been shown to reduce anxiety, depression, and delayed assessment of pain ${ }^{(5)}$. The reported cardiopulmonary effects of MT in adult 
populations include reduction in blood pressure (BP) and heart rate (HR), increase in peak flow and forced vital capacity, and improvement in comfort, pain, anxiety, and tension after cardiac surgery ${ }^{(6-9)}$. There is evidence to suggest that post-exercise massage may be helpful in reducing delayed-onset muscle soreness ${ }^{(10)}$. In addition, Hemmings et al. reported that massage is effective in enhancing perception of recovery after boxing ${ }^{(11)}$, which may lead to improvement in subsequent performance.

Although post-exercise massage has been shown to be beneficial, research on the effects of pre-exercise massage is limited. To date, no studies of pediatric MT have examined the relationship between massage and exercise performance. More specifically, studies evaluating the effect of MT on children's cardiopulmonary function have not been performed.

\section{METHODS}

This prospective pilot study examined the safety and feasibility of measuring the effects of pre-exercise massage on exercise performance and cardiopulmonary response in children with and without heart disease. The Institutional Review Board of the University of California-Los Angeles (UCLA) approved the study protocol.

\section{Participants}

The study cardiologist (RKRC) recruited participants from the pediatric cardiology clinic at the Mattel Children's Hospital at UCLA. All children invited to participate in the study were $6-13$ years of age, ambulatory, and able to ride an exercise bicycle. The study enrolled 16 children, 10 of whom had CHD and a past history of open-heart surgery more than 6 months before enrollment. Of the 6 healthy participants that were enrolled, 3 were siblings of children with CHD, and 3 were recruited from the clinic. This convenience sample of healthy children was included in the study to allow for between-group comparisons. For the healthy participants to be eligible, they had to have a history free of cardiac, pulmonary, or other systematic disease. Participants were given a $\$ 25 \mathrm{gift}$ certificate to Toys'R'Us.

\section{Procedure}

Using an identical protocol (apart from the massage session), each participant underwent two exercise tests: one test with pre-exercise massage, and one test without pre-exercise massage. The interval ("washout period") between the first test and the second test was $2-3$ months to minimize the learning effect from the first test. In addition, the research team's cardiologist judged that this time frame would not have a substantial effect on increased athletic performance resulting from growth and maturation. The order of the tests (with or without massage) was randomized. The study cardiologist, a parent of the child, and a research assistant were present during each test and were not blinded to the child's health condition. The entire procedure (set up, test, and cool down) lasted about 30 minutes.

Exercise tests were conducted in a pediatric exercise laboratory using an electromagnetically braked cycle ergometer suitable for children (Ergometrics 800: SensorMedics, Loma Linda, CA). The 10minute testing procedure was consistent with recommendations from the American Heart Association's Council on Cardiovascular Disease in the Young ${ }^{(12)}$. A 12-lead electrocardiograph and a mouthpiece with a nose clip in place were connected as the children performed a ramp (progressive) protocol to the limit of their endurance. At rest, measures of HR, systolic and diastolic $\mathrm{BP}$, and oxygen uptake $\left(\mathrm{VO}_{2}\right)$ were collected.

After 3 minutes of unloaded pedaling as a warmup, the work rate increased continuously at a rate of 5-15 W/min (depending on a pre-test estimate of each participant's aerobic capacity), with the goal of attaining 8-10 minutes of ramp exercise. The test continued until the participant was unable to keep the pedaling rate above $50 \mathrm{rpm}$, despite standardized verbal encouragement from the study cardiologist. During the exercise protocol, the following peak measures were collected: HR, systolic and diastolic $\mathrm{BP}, \mathrm{VO}_{2}$, anaerobic threshold, work rate, $\mathrm{O}_{2}$ pulse, and minute ventilation. No adverse events occurred, and no test had to be terminated prematurely.

\section{Massage Therapy Intervention}

A volunteer massage therapist (female, certified) with specialized training in pediatric massage provided all of the massage sessions for this study. She was blinded to the health status and group assignment of the participants, treating all children as if they could have had heart disease. Based on a predesigned script, the massage therapist asked the parent if the child had any known allergies. Children were asked if they knew what massage was and where they would and would not like to be touched. Because none of the children had had prior experience with a professional massage therapist, the massage procedure was explained to them. Children were informed that they could stop the massage at any time.

A parent was required to stay in the room with the child throughout the entire 30-minute massage. The 30-minute session was chosen to be consistent with other methods used in pediatric massage and research ${ }^{(4,13)}$. The lighting was dimmed to provide a calm atmosphere, and the massage therapist was encouraged to keep talking to a minimum, with the exception of answering any questions that might arise. No music was used during the session. 
The child wore minimal clothing according to comfort level and was appropriately draped with a sheet. The massage therapist asked verbal permission from the child

- to begin the massage,

- to touch a particular area of the body, and

- to move from one area of the body to a new area of the body.

Gentle-to-moderate pressure was applied during the massage ( $3-4$ on a scale of $1-10$, with 10 being the deepest pressure). A full-body massage was given, excluding the chest. This conscious decision ensured that the therapist would remain blinded to whether the child had undergone heart surgery. The legs were massaged in all children, except in 2 children who preferred to keep their pants on. For those two children, the therapist was able to massage the lower leg up to and including the region just above the knee.

Generally, unless otherwise requested, the child began in a supine position on a standard massage table. The feet and legs were massaged first, followed by the hands and arms, and then the head. The child turned over to a prone position, and the neck, back, and arms were massaged, followed by the legs and feet. The techniques applied included effleurage, petrissage, light percussion, and holds. A light, unscented almond oil was used to perform the massage. The pace, speed, and rhythm of the therapist's application varied according to the child's needs and preferences, and were adjusted throughout the massage session. The massage protocol was designed to be consistent with the practice of pediatric MT, allowing children to make determinations about how and where they are touched throughout the session.

\section{Measurements}

Heart rate was recorded continuously using 12-lead electrocardiography (Cardiosoft: SensorMedics) to evaluate the exercise effort and the endpoint of the test. Heart rate is expected to increase with exercise.

Systolic and diastolic BP were measured every 2 minutes using a manual cuff. During exercise, cardiac output typically rises, resulting in an increase in systolic BP with each stage of the test. Diastolic BP typically remains unchanged during exercise because of the vasodilation that occurs.

For each child, $\mathrm{VO}_{2}$ was recorded for each breath using a mouthpiece with a nose clip. Oxygen uptake typically increases with exercise.

The highest values of peak HR, peak systolic and diastolic $\mathrm{BP}$, and $\mathrm{VO}_{2}$ were recorded. Calculations of peak $\mathrm{VO}_{2}$, ventilatory anaerobic threshold, peak work rate, peak $\mathrm{O}_{2}$ pulse $\left(\mathrm{VO}_{2} / \mathrm{HR}\right)$, and peak minute ventilation were performed using a metabolic measurement cart (Vmax 330: SensorMedics).

\section{Data Analysis}

Each child's age, sex, and health status was recorded. The paired $t$-test was used to determine whether MT had any effect on the various physiologic measures. The unpaired $t$-test was used to compare measures in children with heart disease and those in children showing typical development. All statistical tests were done using the SPSS software package (version 11.0: SPSS, Chicago, IL); a $p$ value of 0.05 was considered statistically significant.

\section{RESULTS}

\section{Participant Characteristics}

Sixteen children (10 boys, 6 girls; mean age: 9.2 \pm 2.2 years) participated in the study. Ten participants had either repaired or palliated CHD. Six participants were healthy children with no known cardiopulmonary or muscular disease. Massage was given to 8 participants $(50 \%)$ on the first visit and to the remaining 8 participants $(50 \%)$ on the second visit. The two visits were spaced $2-3$ months apart.

No adverse event occurred in any exercise test or massage session. No complaints of discomfort were prompted by study participation. No specific technical difficulties occurred during the exercise tests for participants with CHD or for healthy participants. All participants who enrolled in the study completed all study tasks; no participant dropped out.

\section{Resting Measurements}

For resting measurements of $\mathrm{HR}, \mathrm{BP}$, or $\mathrm{VO}_{2}$ with and without pre-exercise massage, we observed no significant differences between participants (Table 1). In this sample, receiving a massage did not seem to affect $\mathrm{HR}, \mathrm{BP}$, and $\mathrm{VO}_{2}$ at rest (before the exercise began).

\section{Exercise Measurements}

For measurements during exercise, children who received pre-exercise massage achieved a significantly higher peak HR [ $6 \mathrm{bpm}(3.45 \%)$ higher], higher peak minute ventilation [5.1 L/min $(12.35 \%)$ higher], and higher peak $\mathrm{VO}_{2}[3.6 \mathrm{~mL} / \mathrm{kg} / \mathrm{min}(10.6 \%)$ higher]. None of the other exercise measures were significantly different when massage was provided before the test (Table 1). To investigate the possibility that these differences could be the result of order effects, we calculated the correlations between the randomly determined ordering data and the individual difference scores for the dependent variables; no correlation was statistically significant (all $p>0.05$ ), which suggests that the observed effects were not likely to have been caused by the order in which children performed the two tests. 
TABLE 1. Mean Physiologic Parameters at Rest and During Exercise for Tests With and Without Pre-exercise Massage

\begin{tabular}{|c|c|c|c|}
\hline \multirow[t]{2}{*}{ Physiologic parameter } & \multicolumn{2}{|c|}{ Preceding massage? } & \multirow{2}{*}{$\begin{array}{c}\mathrm{p} \\
\text { Value }^{a}\end{array}$} \\
\hline & Yes & No & \\
\hline \multicolumn{4}{|l|}{ Resting measures } \\
\hline Heart rate $[\mathrm{HR}(\mathrm{bpm})]$ & $86 \pm 15$ & $86 \pm 12$ & 0.88 \\
\hline $\begin{array}{l}\text { Systolic blood pressure [BP } \\
\quad(\mathrm{mmHg})]\end{array}$ & $94 \pm 10$ & $92 \pm 7$ & 0.45 \\
\hline Diastolic BP (mmHg) & $61 \pm 11$ & $63 \pm 8$ & 0.64 \\
\hline $\mathrm{VO}_{2}(\mathrm{~mL} / \mathrm{kg} / \mathrm{min})$ & $5.7 \pm 1.8$ & $5.7 \pm 1.7$ & 0.94 \\
\hline \multicolumn{4}{|l|}{ Exercise measures } \\
\hline Peak HR (bpm) & $180 \pm 23$ & $174 \pm 21$ & 0.05 \\
\hline Peak systolic BP (mmHg) & $118 \pm 14$ & $119 \pm 13$ & 0.96 \\
\hline Peak diastolic BP (mmHg) & $58 \pm 9$ & $61 \pm 11$ & 0.27 \\
\hline Peak $\mathrm{VO}_{2}(\mathrm{~mL} / \mathrm{kg} / \mathrm{min})$ & $37.3 \pm 11.5$ & $33.7 \pm 12.4$ & $<0.01$ \\
\hline $\begin{array}{l}\text { Anaerobic threshold } \\
(\mathrm{mL} / \mathrm{kg} / \mathrm{min})\end{array}$ & $17.9 \pm 6.3$ & $17.8 \pm \pm 6.7$ & 0.84 \\
\hline Peak work rate (watts) & $69 \pm 44$ & $66 \pm 32$ & 0.43 \\
\hline Peak $\mathrm{O}_{2}$ pulse $\left(\mathrm{VO}_{2} / \mathrm{HR}\right)$ & $7.2 \pm 2.6$ & $6.6 \pm 2.9$ & 0.06 \\
\hline $\begin{array}{l}\text { Peak minute ventilation } \\
(\mathrm{L} / \mathrm{min})\end{array}$ & $46.4 \pm 16.6$ & $41.3 \pm 15.2$ & 0.02 \\
\hline $\begin{array}{l}\text { Respiratory quotient at peak } \\
\mathrm{VO}_{2}\end{array}$ & $1.14 \pm 0.12$ & $1.15 \pm 0.10$ & 0.79 \\
\hline
\end{tabular}

${ }^{\text {a }}$ Based on paired $t$-tests.

A comparison of measurements in children with heart disease and those in healthy participants who received pre-exercise massage showed no significant differences in peak $\mathrm{HR}, \mathrm{BP}$, peak $\mathrm{VO}_{2}$, peak work rate, minute ventilation, or respiratory quotient (Table 2).

\section{DISCUSSION AND CONCLUSIONS}

This study provides evidence for the feasibility and safety of measuring the effects of pre-exercise massage on exercise performance and cardiopulmonary response in children with and without heart disease. None of the children dropped out of the study, which could be a result of the favorable response from the children and their parents and also the gift card incentives. Although the children's responses were not systematically studied, more than one child asked if they could have another massage, and nearly all of the children were smiling after their massage sessions. A few parents asked if they could receive massage, and in some cases, they offered to have their other children participate in the study. Parents at times indicated their surprise at how relaxed their children became during the massage session; they wanted to learn some of the techniques. Many of the massage techniques used for the study could
Table 2. Change in Mean Physiologic Parameters with Massage (Tests With and Without Pre-exercise Massage) for Children With and Without Congenital Heart Disease (CHD)

\begin{tabular}{|c|c|c|c|}
\hline \multirow[t]{2}{*}{ Variable } & \multicolumn{2}{|c|}{$C H D$} & \multirow{2}{*}{$\begin{array}{c}\mathrm{p} \\
\text { Value }^{a}\end{array}$} \\
\hline & Yes & No & \\
\hline Participants $(n)$ & 10 & 6 & \\
\hline \multicolumn{4}{|l|}{ Exercise measures } \\
\hline Peak heart rate [HR (bpm)] & $7.1 \pm 11.7$ & $2.3 \pm 6.9$ & 0.38 \\
\hline $\begin{array}{l}\text { Peak systolic blood pressure } \\
\text { [BP }(\mathrm{mmHg})]\end{array}$ & $2.4 \pm 12.2$ & $-4.3 \pm 7.3$ & 0.24 \\
\hline Peak diastolic BP (mmHg) & $-2.8 \pm 11$ & $-4.0 \pm 12.8$ & 0.84 \\
\hline Peak $\mathrm{VO}_{2}(\mathrm{~mL} / \mathrm{kg} / \mathrm{min})$ & $4.3 \pm 3.1$ & $2.5 \pm 4.1$ & 0.31 \\
\hline $\begin{array}{l}\text { Anaerobic threshold } \\
(\mathrm{mL} / \mathrm{kg} / \mathrm{min})\end{array}$ & $-0.9 \pm 3.6$ & $1.1 \pm 3.7$ & 0.60 \\
\hline Peak work rate (watts) & $5.0 \pm 21$ & $-1 \pm 17$ & 0.59 \\
\hline Peak $\mathrm{O}_{2}$ pulse $\left(\mathrm{VO}_{2} / \mathrm{HR}\right)$ & $0.7 \pm 0.5$ & $0.3 \pm 1.6$ & 0.45 \\
\hline Peak minute ventilation (L/min) & $4.3 \pm 3.0$ & $2.5 \pm 4.1$ & 0.31 \\
\hline Respiratory quotient at peak $\mathrm{VO}_{2}$ & $0.0 \pm 0.1$ & $0.0 \pm 0.2$ & 0.59 \\
\hline
\end{tabular}

${ }^{\text {a }}$ Based on unpaired $t$-tests.

certainly be taught to parents, increasing feasibility of the approach. Parental participation could have a positive effect on the results because children may be more comfortable receiving massage from a parent than from a stranger. In support of the safety of massage, no early terminations of the exercise tests occurred, and no adverse events, injuries, or problems were reported.

It is worth mentioning that children with heart disease showed postural differences that the study's massage therapist noticed, even though she was blinded to the group assignment of the child. The massage therapist and one of the clinical researchers both observed that they seemed to be able to differentiate the children that had heart disease because those children typically drew their shoulders forward and seemed to be "guarding" the chest region by slumping forward. This posture is likely a result of increased muscular tension in these children, especially in the shoulder and chest regions.

In this study, the effects of pre-exercise massage were similar for healthy children and for children with heart disease. Although cardiopulmonary function may be impaired and muscles more deconditioned in children with heart disease, those children seem to benefit from MT as much as healthy children do. A larger clinical study to determine how children with various degrees of cardiopulmonary limitation can benefit from MT to improve their exercise capacity may be useful. Because massage is widely used clinically as part of rehabilitation programs for a variety of conditions, a future study could be conducted within the context of rehabilitation ${ }^{(13)}$. 
Findings from this study indicate that peak HR, peak $\mathrm{VO}_{2}$, and peak minute ventilation were higher when children received massage before exercise testing. Larger studies will be needed to investigate the strength of this finding and to rule out maturation effects. In addition, it would helpful to know how a series of massage sessions over a period of time affect peak $\mathrm{VO}_{2}$. In an adult sample of cancer patients, one supportive study found significant increases in peak $\mathrm{VO}_{2}$ after a multidimensional 6-week exercise intervention that included massage ${ }^{(14)}$. Another pilot study found a $15 \%$ improvement in peak $\mathrm{VO}_{2}$ after 12 weeks of cardiac rehabilitation in children with severe $\mathrm{CHD}^{(15)}$.

Taken together, the results of our study and the evidence from other studies paint an interesting picture of a possible mechanism for the effect of massage on peak $\mathrm{VO}_{2}$. For example, submaximal exercise parameters (such as anaerobic threshold) and resting measures (such as $\mathrm{VO}_{2}$ and $\mathrm{HR}$ ) did not seem to vary between exercise with and without massage. Those findings suggest that, with massage, children exercised at a higher peak level without a change in their aerobic capacity. The possible effect of massage on improved exercise performance appears to be a psychological benefit rather than a direct improvement in cardiopulmonary or muscular physiology. This hypothesis is consistent with previous findings that massage reduces state and trait anxiety levels in children $^{(4)}$. In addition, in a review of the outcomes of CHD, Green reported that psychological issues (including anxiety) are common among adults and children $^{(16)}$. Similarly, Kovacs et al. reported that half the adults with CHD that they interviewed met the diagnostic criteria for a current or past mood or anxiety disorder ${ }^{(17)}$. Future studies that include measurements of anxiety and psychological factors in addition to cardiopulmonary measures are recommended.

\section{CONFLICT OF INTEREST NOTIFICATION}

The authors declare that no conflicts of interest are associated with this research project or publication of findings.

\section{COPYRIGHT}

Published under the CreativeCommons Attribution-NonCommercial-NoDerivs 3.0 License.

\section{REFERENCES}

1. Hoffman JI, Kaplan S. The incidence of congenital heart disease. J Am Coll Cardiol. 2002;39(12):1890-1900.

2. Meberg A, Lindberg H, Thaulow E. Congenital heart defects: the patients who die. Acta Paediatr. 2005;94(8):1060-1065.
3. Costa P, Carriço A, Rêgo C, Areias JC. Exercise testing in pediatric cardiology [Portuguese]. Rev Port Cardiol. 2005;24(6):885-895.

4. Beider S, Moyer CA. Randomized controlled trials of pediatric massage: a review. Evid Based Complement Alternat Med. 2007;4(1):23-34.

5. Moyer CA, Rounds J, Hannum JW. A meta-analysis of massage therapy research. Psychol Bull. 2004;130(1):3-18.

6. Beeken J, Parks D, Cory J, Montopoli G. The effectiveness of neuromuscular release massage therapy in five individuals with chronic obstructive lung disease. Clin Nurs Res. 1998;7(3):309-317.

7. Anderson PG, Cutshall SM. Massage therapy: a comfort intervention for cardiac surgery patients. Clin Nurse Spec. 2007;21(3):161-165.

8. Bauer BA, Cutshall SM, Wentworth LJ, Engen D, Messner PK, Wood CM, et al. Effect of massage therapy on pain, anxiety, and tension after cardiac surgery: a randomized study. Complement Ther Clin Pract. 2010;16(2):70-75.

9. Cutshall SM, Wentworth LJ, Engen D, Sundt TM, Kelly RF, Bauer BA. Effect of massage therapy on pain, anxiety, and tension in cardiac surgical patients: a pilot study. Complement Ther Clin Pract. 2010;16(2):92-95.

10. Hilbert JE, Sforzo GA, Swenson T. The effects of massage on delayed onset muscle soreness. Br J Sports Med. 2003;37(1):72-74.

11. Hemmings B, Smith M, Graydon J, Dyson R. Effects of massage on physiological restoration, perceived recovery, and repeated sports performance. Br J Sports Med. 2000;34(2):109-115.

12. Paridon SM, Alpert BS, Boas SR, Cabrera ME, Caldarera LL, Daniels SR, et al. Clinical stress testing in the pediatric age group: a statement from the American Heart Association Council on Cardiovascular Disease in the Young, Committee on Atherosclerosis, Hypertension, and Obesity in Youth. Circulation. 2006;113(15):1905-1920.

13. Beider S, Mahrer NE, Gold JI. Pediatric massage therapy: an overview for clinicians. Pediatr Clin North Am. 2007;54(6):1025-1041.

14. Adamsen L, Quist M, Midtgaard J, Andersen C, Møller T, Knutsen L, et al. The effect of a multidimensional exercise intervention on physical capacity, well-being and quality of life in cancer patients undergoing chemotherapy. Support Care Cancer. 2006;14(2):116-127.

15. Rhodes J, Curran TJ, Camil L, Rabideau N, Fulton DR, Gauthier NS, et al. Impact of cardiac rehabilitation on the exercise function of children with serious congenital heart disease. Pediatrics. 2005;116(6):1339-1345.

16. Green A. Outcomes of congenital heart disease: a review. Pediatr Nurs. 2004;30(4):280-283.

17. Kovacs AH, Saidi AS, Kuhl EA, Sears SF, Silversides C, Harrison JL, et al. Depression and anxiety in adult congenital heart disease: predictors and prevalence. Int $J$ Cardiol. 2009;137(2):158-64.

Corresponding author: Shay Beider, Integrative Touch for Kids, 8340 N. Thornydale Road, 110-153, Tucson, AZ 85741 USA.

E-mail: shay@integrativetouch.org 